\title{
Role of Hydrogen in Giant Spin Polarization Observed on Magnetic Nanostructures
}

\author{
Werner A. Hofer and Krisztián Palotás \\ Surface Science Research Centre and Department of Physics, University of Liverpool, Liverpool L69 3BX, United Kingdom
}

Stefano Rusponi, Tristan Cren, and Harald Brune

Institute of the Physics of Nanostructures, Ecole Polytechnique Fédérale de Lausanne, Station 3, CH-1015 Lausanne

(Received 22 May 2007; published 17 January 2008)

\begin{abstract}
We demonstrate that the giant spin contrast observed by scanning tunneling microscopy for doublelayer Coislands on $\mathrm{Pt}(111)$ is caused by adsorbates at the apex of the Cr-coated W tip. The most likely candidate, in $a b$ initio simulations, is hydrogen. Here, the electron charge is highly polarized by the adjacent $\mathrm{Cr}$ layers. The hydrogen adsorption site is shown to change from hollow to on top due to the electric field at the tip apex, created by the tunnel voltage.
\end{abstract}

DOI: 10.1103/PhysRevLett.100.026806

One of the currently most active fields in magnetic research is the investigation of the physical density limit of magnetic information storage. The storage medium reaching the highest densities will most likely consist of ferromagnetic monodomain particles with out-of-plane magnetization minimizing dipolar interactions. An additional requirement are uniform magnetic particle properties; i.e., the orientation and absolute value of their moment as well as their anisotropy energies should have narrow distributions. These requirements suggest islands on single crystal surfaces as model systems providing an attractive alternative to chemically self-assembled colloid particles [1,2]. Recently, it has been shown that atomic beam epitaxy of $\mathrm{Co}$ on $\mathrm{Au}(788)$ is capable of producing a particle density of about $26 \mathrm{Tera} / \mathrm{in}^{2}$ with the required uniformity of magnetic properties and absence of dipolar interactions [3]. To improve and to attain insight into these model systems at the atomic scale, spin-polarized scanning tunneling microscopy (SP-STM) [4] is an important complement to spatially integrating measurements. With SPSTM the magnetic properties of individual particles can be observed, e.g., the transition from the blocked to the superparamagnetic state [5], or the spatially and spin-resolved electronic structure of an island [6].

For Co particles grown on Pt(111) SP-STM showed apparent height differences of $\Delta z=20 \pm 5$ pm between islands with up and down magnetization [7]. This contrast in constant-current STM images can be interpreted as magneto-resistance of the tunnel-junction (TMR) for constant gap width [7]

$$
\frac{\Delta R}{R}=\frac{R_{a}-R_{p}}{R_{p}}=\frac{I_{p}}{I_{a}}-1=\exp \left(\frac{1.025 \sqrt{\phi} \Delta z}{\sqrt{e V} 0.1 \mathrm{~nm}}\right)-1,
$$

with $\phi$ typically about $4 \mathrm{eV}$. The measurements translate into a TMR value of $\Delta R / R=50 \pm 15 \%$. However, in some cases much higher TMR values have been observed reaching $\Delta R / R=850 \pm 200 \%$, corresponding to a differ-
PACS numbers: 73.22.-f, 68.37.Ef, 71.10. $-\mathrm{w}, 71.15 . \mathrm{Mb}$

ence in apparent height $\Delta z=110 \mathrm{pm}$. The TMR increase directly translates into an increase of magnetic contrast in the SP-STM measurements. High-resolution atomic scale measurements of magnetic properties can be used to detect effects which are currently below the resolution limit, e.g., canted moments at the island edges expected from measurements of step-decorated vicinal surfaces [8]. It is thus important to determine the origins of this high contrast, since its utilization in a controlled manner will be highly advantageous for the entire field of magnetic research on nanoscale objects.

In this Letter we investigate the reason for this "giant spin-polarization" by theoretical methods and compare the results to new experimental data, obtained by varying the bias-voltage over a wide range for identical tip-conditions. The ground-state electronic structures of the Co islands on $\operatorname{Pt}(111)$ were calculated by first-principles spin-polarized density-functional theory (DFT) [9] implementing an all electron treatment using the projector-augmented wave (PAW) method [10,11]. We considered (i) a double-layer Co island composed of $13 \mathrm{Co}$ atoms (9 first and $4 \mathrm{~s}$ layer) on a flat $\mathrm{Pt}(111)$ surface, and (ii) two pseudomorphic Co layers on $\mathrm{Pt}(111)$. Model (ii) describes well the large island size in the experiment, while model (i) permits to investigate the effect of strain since the low-coordinated atoms have a substantial inward relaxation simulating the overall reduced lattice constant in the Moire pattern by which the islands relieve part of the stress due to the lattice mismatch of $-9.4 \%$ [12]. The SP-STM tip was mimicked by seven and $114 \times 4$ Cr-layers on $\mathrm{W}$ which avoids numerical artifacts due to a double vacuum boundary. On top of the $\mathrm{Cr}$ layers a $\mathrm{Cr}$ adatom simulated the tip apex.

Triangular Co double-layer islands with a diameter of more than $10 \mathrm{~nm}$ and a blocking temperature above $160 \mathrm{~K}$ have been prepared on $\mathrm{Pt}(111)$ by deposition of $0.4 \mathrm{ML}$ at $130 \mathrm{~K}$ and subsequent annealing to $340 \mathrm{~K}$ [7]. Figure 1(a) shows a large scale image with several such islands. The magnetic contrast obtained with a standard Cr-coated tip is $26 \mathrm{pm}$ under the conditions $V_{t}=-80 \mathrm{mV}, I_{t}=0.3 \mathrm{nA}$, 


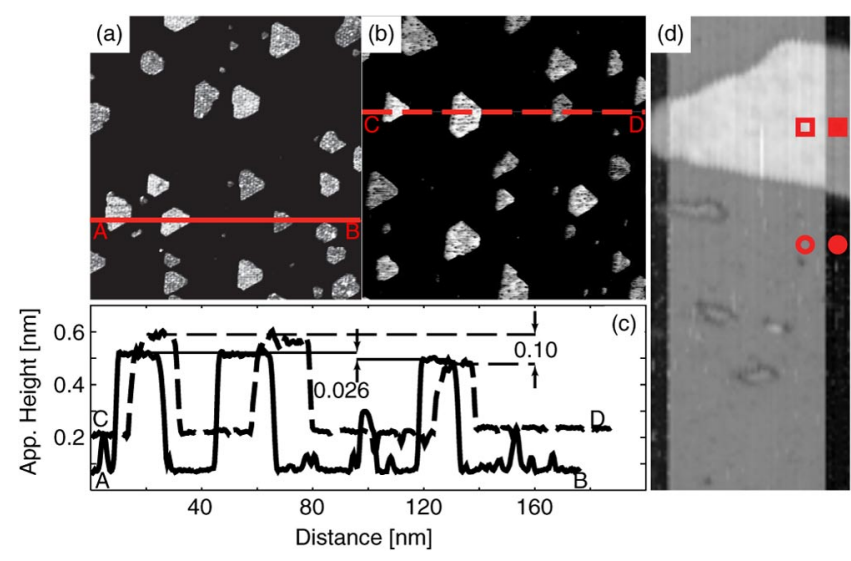

FIG. 1 (color online). Experimental STM constant-current images with low magnetic contrast (a) and high magnetic contrast (b). The line scans across three double-layer Co islands are shown in (c). The Co islands grown on Pt(111) have a narrow size distribution and a diameter in the range of $20-30 \mathrm{~nm}$. (a) The magnetic contrast between different islands, measured with a standard tip, is about $26 \mathrm{pm}$. (b) The magnetic contrast increases to $100 \mathrm{pm}$ with a high spin contrast STM tip. (d) Transition from a low-contrast to a high-contrast tip. The apparent height at the $\mathrm{Pt}$ substrate increases by $0.37 \mathrm{~nm}$ (ciircles), the apparent height of the Co island by $0.21 \mathrm{~nm}$ (squares). The image size of frame (d) is $24 \times 77 \mathrm{~nm}$.

and $T_{\text {sample }}=140 \mathrm{~K}$ [Fig. 1(a)]. Figure 1(b) shows high magnetic contrast of $100 \mathrm{pm}$ [scan lines see Fig. 1(c)]. Images where the spin contrast of the tip changed reversibly reveal for the high spin contrast state larger apparent heights of $0.37 \mathrm{~nm}$ and $0.21 \mathrm{~nm}$ for Pt substrate and Co islands, respectively, [see Fig. 1(d)]. The overall increase of the apparent height in the scans suggests the presence of an additional atom at the apex of the tip.

In addition to the tip described above, we simulated the following trial probes in our calculation of the tunneling current [13]: (i) the adsorbed $\mathrm{Cr}$ was changed to $\mathrm{Co}$, corresponding to material transfer from sample to tip, (ii) we adsorbed $\mathrm{H}, \mathrm{C}$, and $\mathrm{O}$, on top of the $\mathrm{Cr}$ apex atom, or (iii) onto the $\mathrm{Cr}$ surface adjacent to it. (ii) and (iii) correspond to dissociative adsorption of hydrogen, hydrocarbons, and water, being the main constituents in the residual gas, which had a pressure of $3 \times 10^{-11} \mathrm{mbar}$ in the experiments. In addition, we simulated the effect of an electric field during operation of the SP-STM by calculating the ground-state electronic structure of the tip models applying a dipole field of $0.1-0.6 \mathrm{~V} / \mathrm{nm}$ along the surface normal $z$. The tunneling current for every combination of surface and tip was calculated for ferro- and antiferromagnetic configuration of surface and tip. The magnetic contrast at a given bias voltage and current was then determined from the apparent height difference of the constant-current contours, in the very same way as in experiment.

For model (i) we find a Co spin moment between 1.8 and $1.9 \mu_{B}$, in perfect agreement with calculations of Co monolayers on $\mathrm{Pt}(111)$ [14] and with extrapolation from values calculated for smaller monolayer islands equally on $\mathrm{Pt}(111)$ [15]. Analyzing the polarization of $\mathrm{Cr}-$ and Coterminated tip models we find a value of $25 \%$ near the Fermi level. The expected spin contrast using this polarization is $\Delta z=12 \mathrm{pm}$ [see Figs. 2(a) and 2(c)]. This value is only half the experimental value of $26 \mathrm{pm}$ for the lowcontrast tip. However, if we perform a full current simulation with a Cr-terminated tip above a Co island we derive $\Delta z=45 \mathrm{pm}$. If the $\mathrm{Cr}$ apex atom is replaced by Co, this value changes to $35 \mathrm{pm}$. There are two things to be learnt from this result. First the observed contrast with the lowcontrast tip is below the predicted values, which can be caused by the tip magnetization in the experiment being slightly tilted away from out-of-plane direction of the islands, reducing the contrast. The second observation being the higher magnetic contrast of simulations including the STM tip (Cr-tip polarization: $25 \%$, contrast: $46 \mathrm{pm}$ ) as compared to calculations based on the local density alone (tip polarization: $25 \%$, contrast: $12 \mathrm{pm}$ ). This indicates that the local extension of tip states has a major influence on the obtained results $[16,17]$ and it also signifies that precise prediction of the spin contrast requires to go beyond the Julière model.

While substitution of tip atoms by surface atoms plays an important role in other magnetic effects [18], here it cannot account for the giant spin-polarization. Moreover, the large tip-sample distance for the experimental tunnel parameters (we obtain about 0.6-0.7 nm for $-80 \mathrm{mV} / 0.3 \mathrm{nA}$ ) precludes the presence of strong interactions [19]. Therefore the effect can only originate from a modification of the STM tip by nonmetallic adsorbates. It has been shown in the past that, e.g., $\mathrm{CO}$ increases the corrugation of metal surfaces by a factor of 3 [20]. However, the most likely origin of a high tip polarization is the adsorbate polarization induced by the magnetic $\mathrm{Cr}$ surface. This effect is expected to decrease with increasing distance of the tip apex atom from the $\mathrm{Cr}$ surface. Upright standing molecules such as $\mathrm{CO}$ are expected to have smaller polarization than adatoms; therefore we concentrated in the search for potential tips with high spinpolarization on adatoms.

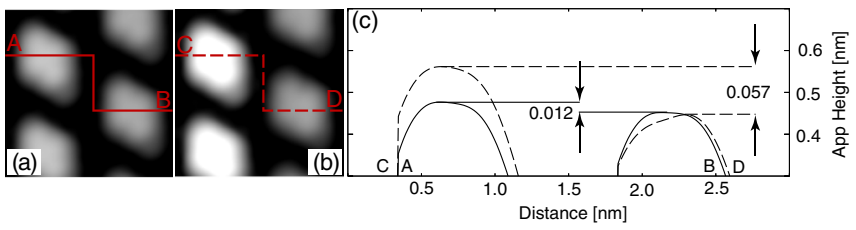

FIG. 2 (color online). Simulated local density contours of double-layer Co islands on $\mathrm{Pt}(111)$ with variable weights of spin-up and spin-down states mimicking different tip polarizations. (a) Polarization 25\%, corresponding to the polarization of a Cr-terminated STM tip. (b) Polarization 95\%. (c) Line scans across two islands with opposite magnetic states. The enhanced polarization increases the magnetic contrast by a factor of 4-5, corresponding to the contrast enahancement observed in the experiments. 
The considered tip models are shown in the top left frames of Fig. 3. The apex atom of the tip is either Co, or $\mathrm{Cr}$. Hydrogen, oxygen, and carbon are adsorbed either on top of the $\mathrm{Cr}$ apex atom, or at the hollow site near the $\mathrm{Cr}$ apex. The latter adsorption site is energetically favored by about $2 \mathrm{eV}$ in all cases. The large energy difference between the two configuration of hydrogen of about $2 \mathrm{eV}$, which is 1 order of magnitude higher than the value obtained for a change of position at a flat metal surface [2123 ], is mainly due to the interaction of the surface dipole at the apex atom [24] with the electronic charge of the underlying surface. This dipole is mostly quenched, if the adsorbate is located near the apex; it is increased, if the adsorbate diffuses to the apex of the tip. Subsequently we calculated the magnetic contrast with these tip models for surface model (ii). We derive a spin moment of $1.88 \mu_{B}$, again in good agreement with former results. The spin contrasts vary dramatically with the chemical nature and the adsorption site of the adsorbates [see Fig. 3(a)]. With one exception, $\mathrm{C}$ adsorbed at the hollow site, the magnetic contrast is reduced, or even vanishes for the ground-state adsorption sites. By contrast, adsorption of all three atoms at the $\mathrm{Cr}$ apex leads to a substantial increase of the magnetic contrast, compared to a clean or Co-covered tip. In case of hydrogen the obtained value of $100 \mathrm{pm}$ is in excellent agreement with experimental data. The fact that giant spin contrasts are obtained only very rarely, and only for a relatively short period in the experiment, indicates that $\mathrm{C}$ atoms are most likely not the cause since they would lead to stable high-contrast tips. The physical origin of the contrast changes is found in the chemical bonding of the adsorbates to the $\mathrm{Cr}$ apex atom. For $\mathrm{Cr}$, which is antiferromagnetic, the bonding of $\mathrm{Cr}$ atoms to adjacent layers is the key parameter in its magnetic structure. Changing the bonding structure, e.g., by coadsorption of adsorbates at the $\mathrm{Cr}$ apex, can thus have a profound influence on magnetic properties. To determine the influence of adsorbates on the tip apex, we integrated the density of states (DOS) over a sphere around the $\mathrm{Cr}$ apex atom. In Fig. 3(b) we show the polarization, derived from the partial DOS integrated over the $\mathrm{Cr}$ apex atom (PDOS). It can be seen that the polarization is quenched for $\mathrm{O}_{S}$ and $\mathrm{H}_{S}$, it is substantially increased over a wide energy range for $\mathrm{H}_{T}$. Qualitatively, the result is in agreement with the results for the magnetic contrast [Fig. 3(a)]. However, the agreement is only qualitative, since the decay length of spinpolarized states and their overlap with surface states does not enter the PDOS.

Dynamic processes become increasingly recognized to play a key role in STM experiments. The most obvious example is inelastic tunneling spectroscopy [25], where tunneling electrons excite surface phonons or molecular vibrations [26]. However, also spin flip processes are due to inelastic events and give rise to significantly higher conductance steps [27]. In our case, given the dramatic contrast changes with adsorbate position the most likely dynamic change is expected to happen at the tip. Therefore we considered the effect of electric fields created by the tunnel bias on the energy of the adsorption sites. The effect of an applied external field predominantly changes the total energy of the system in two ways: the field-ion interaction is linear with the field and negative; the band energy, or the subsequent reaction of the electron density is positive. The contributions for different field intensities and for two tip geometries are shown in Fig. 4(a) and 4(b). It can be seen that the $\mathrm{H}$ on top position becomes more favorable at a field intensity of about $130 \mathrm{mV} / \mathrm{nm}$ [Fig. 4(c)]. Given that the changes in the experiments occur at about $100 \mathrm{mV}$ for a distance of $0.6 \mathrm{~nm}$, the simulated results are in good agreement with experimental values. This suggests that the hydrogen atom may indeed in the experiment diffuse onto the $\mathrm{Cr}$ apex atom if the bias voltage is high enough. The very rare occurrence of the effect and its instability are entirely consistent with the

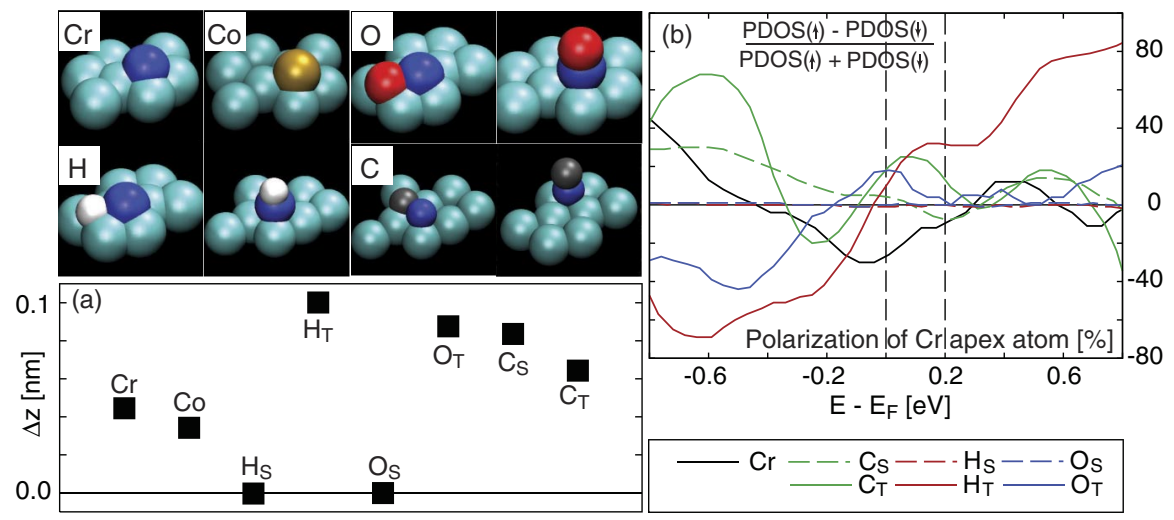

FIG. 3 (color online). SP-STM tip modified by adsorbed atoms, magnetic contrast at $V_{t}=-80 \mathrm{mV}, I_{t}=0.3 \mathrm{nA}$ (a), and polarization of the $\mathrm{Cr}$ apex atom with different adsorbates and configurations (b). (Top left frames) Tip with $\mathrm{Cr}$, Co apex, and modified by $\mathrm{C}, \mathrm{O}$, and $\mathrm{H}$ adsorbed at the apex, or at the surface. (a) Magnetic contrast of modified tips. The highest contrast of about $100 \mathrm{pm}$, is obtained with a hydrogen atom adsorbed at the apex. (b) The polarization obtained from the partial DOS integrated over the $\mathrm{Cr}$ apex atom (see expression). It can be seen that the polarization agrees qualitatively with the obtained magnetic contrast; it vanishes for $\mathrm{H}_{S}$ and $\mathrm{O}_{S}$, and it is a maximum for nearly the whole energy range for $\mathrm{H}_{T}$. 


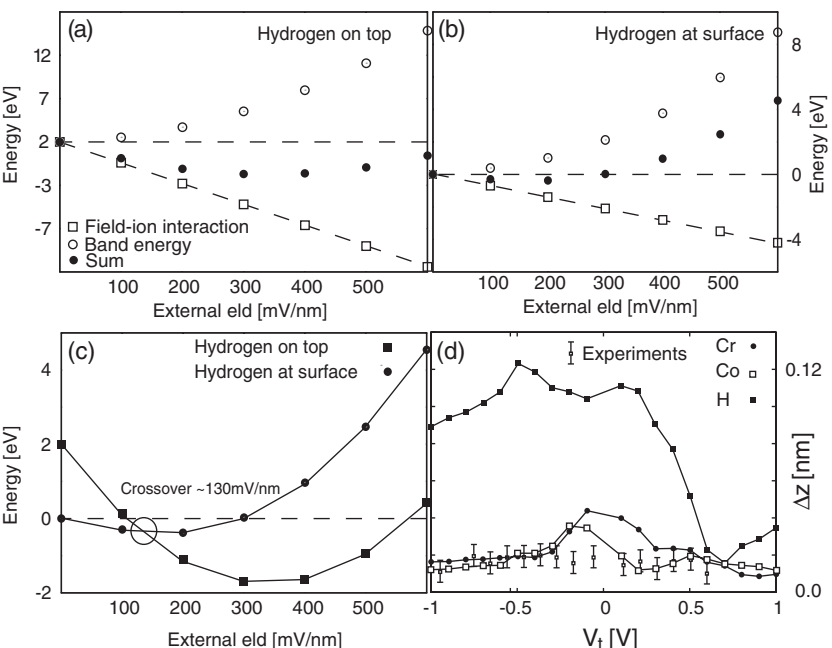

FIG. 4. Field dependence of ion-field interactions (empty squares), band energy (empty circles), and change of total energy (full circles) for hydrogen adsorbed on top (a) and hydrogen at the surface hollow site (b). Note the linearity of the field-ion interaction energy (dashed lines). (c) With increasing field the top position (squares) becomes more favorable, winning beyond $130 \mathrm{mV} / \mathrm{nm}$ against the hollow site (circles). (d) Bias dependence of magnetic contrast. For the entire bias interval the contrast of a clean Cr- or a Co-terminated tip is in agreement with experiment. For a hydrogen-terminated tip the contrast remains in the range of $100 \mathrm{pm}$ for $-0.8 \mathrm{~V} \leq V_{t} \leq 0.2 \mathrm{~V}$.

theoretical finding that the giant spin polarization is due to hydrogen adsorption and a dynamic change in adsorption site due to the external field between the surface and the STM tip. A similar behavior is not observed for oxygen, presumably due to the smaller induced dipole moments of $\mathrm{O}$ compared with $\mathrm{H}$.

It is interesting to note that the effect of hydrogen has quite frequently been discussed, and that it was qualitatively inferred that the small region occupied by the hydrogen electron could in fact lead to an enhancement of STM contrast. Here, we find that spin-polarization is enhanced by a factor of 5 , which leads to an increase of the TMR by more than an order of magnitude. The high magnetic contrast of a hydrogen-terminated $\mathrm{Cr}$ tip is due to the high polarization of the hydrogen electron. At the Fermi level the polarization is about $80 \%$. We may thus conclude that a high polarizability as well as a high mobility under external fields are the essential ingredients for obtaining very high magnetic contrast. These conditions are extremely well met for atomic hydrogen. From a practical point of view it suggests to investigate $\mathrm{H}$ adsorption also in planar TMR junctions [28-30].

A key figure of merit in magnetic tunnel junctions is the bias range over which a given TMR is observed. In Fig. 4(d) we compare experiments with a standard tip for a bias range from $-1 \mathrm{~V}$ to $0.6 \mathrm{~V}$ with simulated results for $\mathrm{Co}$ and $\mathrm{Cr}$ as well as $\mathrm{H}$ terminated tips. It can be seen that the magnetic contrast $\Delta z$ in the experiments remains close to $20 \mathrm{pm}$ over the whole range. Simulations with a $\mathrm{Cr}-$, or
Co-terminated tip are in perfect agreement with this behavior for low negative bias $(<-0.2 \mathrm{~V})$ and for high positive bias $(>0.2 \mathrm{~V})$. The discrepancy of about 10 $20 \mathrm{pm}$ in the low bias regime is most likely due to an incomplete alignment of surface and tip magnetization. Since it was assumed in the simulation that the magnetization vectors are parallel, a small angle between the vectors will lead to substantial reductions of the magnetic contrast $[17,18]$. Simulating the magnetic contrast of a hydrogen-terminated tip we find that it is above $100 \mathrm{pm}$ in a wide bias range of $-0.8 \mathrm{~V} \leq V_{t} \leq 0.2 \mathrm{~V}$. Above $+0.2 \mathrm{~V}$ the value decreases to about $20 \mathrm{pm}$.

[1] The Physics of Ultra-High Density Magnetic Recording, Springer Series in Surface Science Vol. 41, edited by M. L. Plumer, J. van Ek, and D. Weller (Springer, Berlin, 2001).

[2] C. Petit, A. Taleb, and M. P. Pileni, J. Phys. Chem. B 103, 1805 (1999).

[3] N. Weiss et al., Phys. Rev. Lett. 95, 157204 (2005).

[4] R. Wiesendanger et al., Phys. Rev. Lett. 65, 247 (1990).

[5] M. Bode, O. Pietzsch, A. Kubetzka, and R. Wiesendanger, Phys. Rev. Lett. 92, 067201 (2004).

[6] O. Pietzsch et al., Phys. Rev. Lett. 96, 237203 (2006).

[7] S. Rusponi et al., Appl. Phys. Lett. 87, 162514 (2005).

[8] P. Gambardella et al., Phys. Rev. Lett. 93, 077203 (2004).

[9] G. Kresse and J. Furthmüller, Phys. Rev. B 54, 11169 (1996).

[10] G. Kresse and D. Joubert, Phys. Rev. B 59, 1758 (1999).

[11] J. P. Perdew et al., Phys. Rev. B 46, 6671 (1992).

[12] T. Cren et al., J. Phys. Chem. B 108, 14685 (2004).

[13] K. Palotas and W. A. Hofer, J. Phys. Condens. Matter 17, 2705 (2005).

[14] R. Wu, C. Li, and A. J. Freeman, J. Magn. Magn. Mater. 99, 71 (1991).

[15] P. Gambardella et al., Science 300, 1130 (2003).

[16] W. A. Hofer et al., Surf. Sci. Lett. 405, L514 (1998).

[17] W. A. Hofer, Prog. Surf. Sci. 71, 147 (2003).

[18] W. A. Hofer and A. J. Fisher, J. Magn. Magn. Mater. 267, 139 (2003).

[19] W. A. Hofer, A. Garcia-Lekue, and H. Brune, Chem. Phys. Lett. 397, 354 (2004).

[20] J. R. Hahn and W. Ho, Phys. Rev. Lett. 87, 196102 (2001).

[21] D. C. Ford, Y. Xu, and M. Mavrikakis, Surf. Sci. 587, 159 (2005).

[22] T. Mitsui et al., Angew. Chem., Int. Ed. Engl. 119, 5859 (2007).

[23] C. Klein et al., Phys. Rev. Lett. 90, 176101 (2003).

[24] L. Limot et al., Phys. Rev. Lett. 94, 126102 (2005).

[25] N. Lorente, M. Persson, L. J. Lauhon, and W. Ho, Phys. Rev. Lett. 86, 2593 (2001).

[26] N. Lorente, Appl. Phys. A: Mater. Sci. Process. 78, 799 (2004).

[27] C. F. Hirjibehedin, C. P. Lutz, and A. J. Heinrich, Science 312, 1021 (2006).

[28] K. Wang, P. M. Levy, S. Zhang, and L. Szunyogh, Philos. Mag. 83, 1255 (2003).

[29] W. H. Butler and A. Gupta, Nat. Mater. 3, 845 (2004).

[30] X. G. Zhang and W. H. Butler, Phys. Rev. B 70, 172407 (2004). 